\section{Seleção de medicamentos essenciais e a carga de doença no Brasil}

\author{
Selection of essential medicines and the \\ burden of disease in Brazil
}

\section{Selección de medicamentos esenciales y carga de las enfermedades en Brasil}

\author{
${ }^{1}$ Instituto de Tecnologia \\ em Fármacos, Fundação \\ Oswaldo Cruz, Rio de Janeiro, \\ Brasil. \\ 2 Escola Nacional de Saúde \\ Pública Sergio Arouca, \\ Fundação Oswaldo Cruz, \\ Rio de Janeiro, Brasil. \\ Correspondência \\ T. A. Figueiredo \\ Centro Tecnológico de \\ Medicamentos, Instituto de \\ Tecnologia em Fármacos, \\ Fundação Oswaldo Cruz. \\ Av. Comandante Guaranys \\ 447, Rio de Janeiro, $R J$ \\ 22775-903, Brasil. \\ tatianafigueiredo@far. \\ fiocruz.br
}

\begin{abstract}
The World Health Organization (WHO) defines essential medicines as those that meet the population's priority healthcare needs. Their selection aims to reflect collective needs, thus recommending the use of studies on global burden of disease. An exploratory study was performed to link the medicines from the RENAME lists to Global Burden of Disease in Brazil (1998 and 2008) and the scientific evidence. The study thus sought to verify whether the RENAME (2002 to 2012) met WHO guidelines for drug selection. Although RENAME 2010 and 2012 both adhere to Global Burden of Disease in Brazil 2008, the 2012 version includes a longer list of medicines and appears to be pressured by the growing market for new technologies. Thus, RENAME is no longer a list of essential medicines, but has become a list of financing for pharmaceutical care.
\end{abstract}

Essential Drugs; Burden of Disease; Pharmaceutical Services
Tatiana Aragão Figueiredo 1 Joyce Mendes de Andrade Schramm 2 Vera Lúcia Edais Pepe ${ }^{2}$

\section{Resumo}

Os medicamentos essenciais são definidos pela Organização Mundial da Saúde (OMS) como aqueles que satisfazem as necessidades prioritárias de cuidado de saúde da população. Sua seleção busca refletir necessidades coletivas, recomendando-se, para tanto, a utilização dos estudos de carga global de doença. Foi realizado um estudo exploratório relacionando os medicamentos das RENAME aos estudos de Carga Global de Doença no Brasil, de 1998 e 2008, e às evidências científicas. Buscou-se, assim, verificar se os elencos de medicamentos nas RENAME (2002 a 2012) atenderam às recomendações da OMS para seleção de medicamentos. Embora as RENAME 2010 e 2012 acatem semelhantemente à Carga Global de Doença Brasil 2008, a versão de 2012 o faz com maior número de medicamentos, parecendo ter sido tensionada por pressões do crescente mercado de novas tecnologias. Dessa forma, a RENAME deixou de ser uma relação de medicamentos essenciais, tornando-se uma lista de financiamento da assistência farmacêutica.

Medicamenos Essenciais; Carga da Doença; Assistência Farmacêutica 


\section{Introdução}

A Política Nacional de Medicamentos (PNM) tem como um de seus objetivos garantir o acesso da população aos medicamentos considerados essenciais 1. A adoção da Relação Nacional de Medicamentos Essenciais (RENAME) é uma das diretrizes e prioridades da PNM, constituindo-se um dos eixos estratégicos da Política Nacional de Assistência Farmacêutica (PNAF) 2.

Medicamentos essenciais são definidos pela Organização Mundial da Saúde (OMS) como aqueles que satisfazem as necessidades prioritárias de cuidado de saúde da população ${ }^{3}$. A seleção dos medicamentos essenciais se pauta na perspectiva epidemiológica e busca refletir necessidades coletivas, sendo recomendada pela OMS a utilização dos estudos de carga global de doença para identificação dos problemas de saúde da população ${ }^{3}$. A carga global de doença, por sua vez, utiliza o indicador DALY (disability adjusted life of years - anos de vida perdidos Ajustados por Incapacidade). O DALY é a soma dos anos de vida perdidos devidos à morte prematura (YLL - years of life lost) e dos anos vividos com incapacidade (YLD - years lived with disability). Logo, acrescenta aos indicadores clássicos de mortalidade e morbidade o peso relativo de uma determinada doença em termos de anos de vida perdidos (em relação à expectativa de vida na referida população) ajustados por incapacidades. Ao identificar morbidades e respectivas sequelas comprometedoras da qualidade de vida dos indivíduos, o DALY é capaz de identificar as doenças que não levam ao óbito, mas que são incapacitantes 4,5 .

Além disso, a seleção de medicamentos essenciais deve utilizar os critérios de eficácia, segurança, conveniência, qualidade e custo-efetividade ${ }^{3}$. Essa etapa busca, ainda, ser um instrumento orientador da prática, a fim de melhorar a qualidade da atenção à saúde, a gestão dos medicamentos, a capacitação dos prescritores e a informação dos cidadãos 6,7 .

A proposta de seleção desses medicamentos é um exercício de inteligência clínica e de gestão, não sendo excluídos, dessa forma, os medicamentos de alto custo, desde que representem a melhor escolha para uma condição epidemiologicamente relevante 6,7,8. Segundo Hogerzeil 7, os medicamentos essenciais não são medicamentos de segunda categoria selecionados para os pobres, mas sim o tratamento mais custo-efetivo para uma dada condição patológica, mostrando que, no processo de seleção, é igualmente importante ponderar os custos e os benefícios da administração de um novo tratamento, em relação a outro já existente 9 .
Os critérios de seleção de medicamentos, nos últimos anos, deixaram de se pautar apenas em uma abordagem orientada por experiência clínica, e desde a versão de 2002, a RENAME tem utilizado a Medicina Baseada em Evidências para sua elaboração 6,10. Além disso, no Brasil, a inclusão na RENAME, até sua versão 2010, considerou os medicamentos recomendados até a segunda linha de tratamento 10 .

As listas nacionais de medicamentos essenciais são utilizadas para orientar a aquisição, distribuição e a produção nacional de medicamentos 6 . Devem, portanto, ser um instrumento orientador das ações de planejamento e de organização da assistência farmacêutica no Sistema Único de Saúde (SUS) 9, sendo a RENAME a base para a organização das listas estaduais e municipais 1,2 .

No Brasil, a atualização da RENAME seguiu a orientação da OMS no período de 2000 a 2010, com cinco revisões. A RENAME 2012 apresentou mudanças, inclusive na definição de medicamentos essenciais. Segundo a Resolução no 1, de 17 de janeiro de 2012, que estabelece as diretrizes nacionais da RENAME 2012, medicamentos essenciais são aqueles definidos pelo SUS para garantir o acesso do usuário ao tratamento medicamentoso 11. A atual RENAME é constituída por todos os medicamentos constantes nos Componentes de Financiamento da Assistência Farmacêutica (Componente Básico - CBAF, Componente Estratégico - CESAF e Componente Especializado - CEAF), sendo também incluída a Relação Nacional de Insumos Farmacêuticos e a Relação Nacional de Medicamentos de Uso Hospitalar. Nela também foram inseridos os medicamentos fitoterápicos e homeopáticos. Só não constam nessa última versão os medicamentos de uso exclusivo na oncologia, os oftalmológicos não presentes no CBAF e os de uso em urgências e emergências, presentes na Tabela de Procedimentos, Órteses, Próteses e Medicamentos do SUS da Relação Nacional de Ações e Serviços de Saúde (RENASES) 12.

Em consonância com a orientação da OMS de utilizar a carga global de doença como base para a identificação dos problemas de saúde da população para seleção do elenco de medicamentos da RENAME, este artigo tem como objetivo verificar em que medida os elencos de medicamentos das revisões da RENAME atenderam, por um lado, às principais causas responsáveis pela carga de doença no Brasil, evidenciadas nos estudos de 1998 (Gadelha AMJ, Leite IC, Valente JG, Schramm JMA, Portela MC, Campos MR, et al. Relatório Final do Projeto Estimativa da Carga de Doenças do Brasil, 1998. Rio de Janeiro: Escola Nacional de Saúde Pública, Fundação Oswaldo 
Cruz; 2002) e 2008 (Leite IC, coordenador. Relatório Final. Carga de Doenças do Brasil, 2008. Rio de Janeiro: Escola Nacional de Saúde Pública Sergio Arouca, Fundação Oswaldo Cruz/Núcleo de Pesquisa em Métodos Aplicados aos Estudos de Carga Global de Doença; 2013), e, por outro lado, às recomendações da OMS no que diz respeito à seleção de medicamentos baseada em evidências científicas.

\section{Métodos}

Foi realizado um estudo exploratório que relaciona o perfil dos medicamentos selecionados nas cinco últimas versões da RENAME aos resultados dos estudos de Carga Global de Doença do Brasil (1998 e 2008) e às evidências científicas existentes na base de dados da Micromedex (Thomson Reuters Healthcare Community. DrugDex System. http: / /www.micromedex.com/ products/drugdex/, acessado em 27/Mai/2013).

\section{Fonte de dados}

Na definição das causas mais relevantes, utilizaram-se os estudos de Carga Global de Doença no Brasil, realizados para os anos de 1998 e 2008.

Nos estudos de Carga Global de Doença Brasil, as doenças (ou causas) são reunidas em três grandes grupos 13 . O primeiro refere-se às doenças transmissíveis, às causas maternas, perinatais e nutricionais; o segundo, às doenças não transmissíveis; o terceiro, às causas externas.

Num segundo nível, cada grande grupo se divide em grupos de doenças. Por exemplo, o grupo "doenças não transmissíveis" contempla 14 subgrupos de doenças, incluindo neoplasias malignas e diabetes mellitus. Por fim, no terceiro nível, esses grupos de doenças se subdividem em doenças específicas. Por exemplo, "neoplasia maligna" é subdividida em 17 tipos de câncer, cuja classificação é realizada pela CID10 (Classificação Internacional de Doenças, 10a revisão).

Neste estudo, as anomalias congênitas e as causas externas foram excluídas, com exceção da classificação "outras causas externas não intencionais”, em razão da impossibilidade de lhes atribuir medicamentos específicos. Para "outras causas externas não intencionais", foram considerados os soros antiofídicos. Excluíram-se ainda as causas cujo tratamento seja cirúrgico e/ou não possua medicamento específico, como baixo peso ao nascer, catarata, obstrução de trabalho de parto, apendicite, asfixia e traumatismo ao nascer. Assim, para o Projeto Carga de Doença 1998, foram consideradas 94 causas de DALY e, para o estudo Carga Global de Doença do Brasil 2008, foram consideradas 85 causas de DALY.

Convém ainda esclarecer que a RENAME é constituída por fármacos, imunoterápicos e correlatos, entretanto, para facilitar a leitura do texto, tudo foi denominado como medicamento. Portanto, para identificação dos medicamentos, utilizaram-se as versões da RENAME 2002, 2006, 2008, 2010 e 2012.

Por sua vez, a busca de evidência científica utilizou como base de dados o Thomsom Micromedex-DrugDex System.

\section{Plano de análise}

Os medicamentos foram classificados segundo a Anatomical Therapeutic Chemical Classification (ATC) 14. Como a classificação ATC considera a principal indicação terapêutica, um mesmo medicamento pode possuir mais de uma ATC, dependendo da apresentação farmacêutica e do uso terapêutico correspondente. Foi considerada, então, a ATC segundo a apresentação selecionada nas RENAME.

Foram excluídos os medicamentos que não possuem ATC, como os medicamentos fitoterápicos e os testes diagnósticos, e ainda aqueles sem causa de DALY correspondente, como medicamentos para anestesia, veículo de diluição, antídoto, medicamentos para contracepção, medicamentos para hidratação ou medicamentos para tratamento paliativo.

Os medicamentos foram correlacionados às indicações terapêuticas principais, considerando: (1) a classificação ATC; (2) as indicações do Formulário Terapêutico Nacional da Rename e do registro sanitário da Anvisa; (3) as indicações terapêuticas dos Protocolos Clínicos e Diretrizes Terapêuticas do Ministério da Saúde; (4) as indicações terapêuticas contidas na base de dados Thomsom Micromedex - Drugdex System. Por fim, em caso de dúvida, foi consultado um profissional especialista.

A análise da adequação do medicamento à carga global de doenças foi realizada em três etapas. Na primeira, verificou-se a existência de ao menos um medicamento do elenco das RENAME para cada causa de DALY. Nesse caso, a causa foi classificada como causa de DALY atendida. Assim, foram relacionados o segundo nível da carga global de doença - referente aos grupos de doenças - e o quarto nível da ATC subgrupo terapêutico/farmacológico/químico. As versões da RENAME 2002, 2006 e 2008 foram comparadas com o estudo da carga global de doenças de 1998, e os elencos da RENAME 2010 e 2012 foram comparados com o estudo da Carga Global de Doença de 2008. 
Na segunda etapa, conferiu-se o número de medicamentos existentes em cada elenco das RENAME para as 15 primeiras causas de DALY. Nessa etapa, foram relacionados o terceiro nível da GBD, correspondente às doenças específicas, e o quinto nível da ATC.

Já na terceira etapa, verificaram-se a presença na lista de medicamentos essenciais da OMS 15 e as evidências científicas existentes para os medicamentos incluídos na RENAME 2012 e não presentes na Rename 2010, considerando as 15 principais causas de DALY do estudo Carga Global de Doença 2008.

Empregou-se a base de dados Thomsom $\mathrm{Mi}$ cromedex - DrugDex System para classificação da força da evidência, força de recomendação e eficácia. A busca foi realizada pelo fármaco, pois a pesquisa pelo medicamento em sua respectiva apresentação não é possível nesta base de dados. Dessa forma, pelo documento completo do fármaco, no item "usos terapêuticos", examinaram-se a eficácia, a força da evidência e a força de recomendação para o uso terapêutico referente à causa de DALY do estudo Carga de Doença do Brasil 2008.

A força da evidência se divide em: Categoria A - evidência baseada em metanálises de estudos clínicos randomizados controlados homogêneos, com desfechos e graus de resultados entre os estudos individuais. Compreende estudos múltiplos com ensaios clínicos bem realizados, com controle e randomizados, envolvendo grande número de pacientes; Categoria $\mathrm{B}$ - evidência baseada em informações obtidas de metanálises de ensaios controlados randomizados, com conclusões conflitantes no que se refere ao desfecho e graus de resultados entre os estudos individuais. Compreende ensaios controlados randomizados que envolveram pequeno número de pacientes ou tinham falhas metodológicas significantes (por exemplo, viés, imprecisão de análise etc.) e estudos não randomizados (por exemplo, estudos de coorte, estudos de casocontrole, estudos observacionais); Categoria C evidência baseada em dados obtidos de opinião de especialista ou consenso, relato de caso ou série de casos.

A força de recomendação se divide em: Classe I (recomendado) - determinado teste ou tratamento demonstrou-se útil e deveria ser empregado; Classe IIa (recomendado na maioria dos casos) - determinado teste ou tratamento é, geralmente, considerado útil e indicado na maioria dos casos; Classe IIb (recomendado em alguns casos) - determinado teste ou tratamento pode ser útil e é indicado em alguns, mas não na maioria dos casos; Classe III (não recomendado) - determinado teste ou tratamento não é útil e deve ser evitado; Classe indeterminada (evidência inconclusiva).

Já a eficácia, segundo o Thomsom Micromedex - DrugDex System, é classificada em: Classe I (eficaz) - evidência e/ou opinião de especialistas sugere que um determinado tratamento medicamentoso é eficaz para uma indicação específica; Classe IIa (evidência favorece eficácia) - evidência e/ou opinião de especialistas é conflitante sobre se um determinado tratamento medicamentoso para uma indicação específica é eficaz, mas o peso da evidência e/ou opinião de especialista favorece eficácia; Classe IIb (evidência é inconclusiva) - evidência e/ou opinião de especialistas é conflitante sobre se um determinado tratamento medicamentoso para uma indicação específica é eficaz, mas o peso da evidência e/ou opinião de especialista argumenta contra a eficácia; Classe III (ineficaz) - evidência e/ou opinião de especialistas sugere que um determinado tratamento medicamentoso para uma indicação específica seja ineficaz.

\section{Resultados e discussão}

A RENAME 2002 contém 375 medicamentos; as versões de 2006 e 2008 apresentam 349 e 363, respectivamente. Essas três versões atenderam, com ao menos um medicamento, igualmente a 86 das 94 causas de DALY (91\%) presentes no estudo Carga de Doença do Brasil 1998. Dentre as causas atendidas, 36 (42\%) referem-se ao Grupo I - doenças infecciosas e parasitárias, maternas, perinatais e nutricionais, $49(57 \%)$ ao Grupo II - doenças não transmissíveis e $1(0,7 \%)$ à única causa considerada do Grupo III - causas externas.

A verificação da existência de ao menos um medicamento no elenco das Rename para cada causa de DALY, organizada pelo segundo nível, apresentou resultado satisfatório. Apenas as doenças infecciosas e parasitárias, câncer, deficiências nutricionais, doenças geniturinárias e condições orais não foram atendidas na totalidade (Tabela 1).

A RENAME 2010 compreende 376 medicamentos; a versão de 2012, 440. Ambas atenderam, respectivamente, a 73 (86\%) e 74 (87\%) das 85 causas de DALY presentes no estudo de Carga de Doença do Brasil 2008. Das causas atendidas, $39,5 \%$ são referentes ao Grupo I - doenças infecciosas e parasitárias, maternas, perinatais e nutricionais, $60 \%$ são referentes ao Grupo II - doenças não transmissíveis e $0,5 \%$, à única causa considerada do Grupo III - causas externas.

Na identificação de medicamentos no elenco das RENAME, para cada causa de DALY, organizada pelo segundo nível, as versões de 2010 e 
Tabela 1

Causas de DALY do estudo Carga de Doença do Brasil 1998 atendidas pelas RENAME 2002, 2006 e 2008, por grupo de causas.

\begin{tabular}{|c|c|c|c|c|c|c|}
\hline \multirow[t]{3}{*}{ Causas (2o nível) } & \multicolumn{6}{|c|}{ RENAME } \\
\hline & \multicolumn{2}{|l|}{2002} & \multicolumn{2}{|l|}{2006} & \multicolumn{2}{|l|}{2008} \\
\hline & $\begin{array}{l}\text { Número de causas } \\
\text { atendidas/Número de } \\
\text { causas existentes nos } \\
\text { grupos }\end{array}$ & $\%$ & $\begin{array}{l}\text { Número de causas } \\
\text { atendidas/Número de } \\
\text { causas existentes nos } \\
\text { grupos }\end{array}$ & $\%$ & $\begin{array}{l}\text { Número de causas } \\
\text { atendidas/Número de } \\
\text { causas existentes nos } \\
\text { grupos }\end{array}$ & $\%$ \\
\hline Doenças neuropsiquiátricas & $13 / 13$ & 100 & $13 / 13$ & 100 & $13 / 13$ & 100 \\
\hline Condições durante o período perinatal & $2 / 2$ & 100 & $2 / 2$ & 100 & $2 / 2$ & 100 \\
\hline Desordens dos órgãos dos sentidos & $2 / 2$ & 100 & $2 / 2$ & 100 & $2 / 2$ & 100 \\
\hline Doenças respiratórias crônicas & $3 / 3$ & 100 & $3 / 3$ & 100 & $3 / 3$ & 100 \\
\hline Doenças aparelho digestivo & $3 / 3$ & 100 & $3 / 3$ & 100 & $3 / 3$ & 100 \\
\hline Infecções respiratórias & $3 / 3$ & 100 & $3 / 3$ & 100 & $3 / 3$ & 100 \\
\hline Doenças musculoesqueléticas & $3 / 3$ & 100 & $3 / 3$ & 100 & $3 / 3$ & 100 \\
\hline Causa externa não intencional & $1 / 1$ & 100 & $1 / 1$ & 100 & $1 / 1$ & 100 \\
\hline Condições maternas & $5 / 5$ & 100 & $5 / 5$ & 100 & $5 / 5$ & 100 \\
\hline Diabetes mellitus & $1 / 1$ & 100 & $1 / 1$ & 100 & $1 / 1$ & 100 \\
\hline Doenças de pele & $1 / 1$ & 100 & $1 / 1$ & 100 & $1 / 1$ & 100 \\
\hline Doenças endócrinas/metabólicas & $1 / 1$ & 100 & $1 / 1$ & 100 & $1 / 1$ & 100 \\
\hline Doenças infecciosas e parasitárias & $23 / 24$ & 96 & $23 / 24$ & 96 & $23 / 24$ & 96 \\
\hline Doenças cardiovasculares & $5 / 5$ & 100 & $5 / 5$ & 100 & $5 / 5$ & 100 \\
\hline Câncer & $13 / 17$ & 77 & $13 / 17$ & 77 & $13 / 17$ & 77 \\
\hline Deficiências nutricionais & $3 / 4$ & 75 & $3 / 4$ & 75 & $3 / 4$ & 75 \\
\hline Doenças geniturinárias & $3 / 4$ & 75 & $3 / 4$ & 75 & $3 / 4$ & 75 \\
\hline Condições orais & $1 / 2$ & 50 & $1 / 2$ & 50 & $1 / 2$ & 50 \\
\hline Total & $86 / 94$ & 91 & $86 / 94$ & 91 & $86 / 94$ & 91 \\
\hline
\end{tabular}

DALY: disability adjusted life of years; RENAME: Relação Nacional de Medicamentos Essenciais.

2012 apresentaram algumas diferenças em relação às RENAME anteriores. A RENAME 2012, além das causas já atendidas pela versão de 2010, também atende totalmente às doenças neurológicas e deficiências nutricionais (Tabela 2). O atendimento completo das doenças neurológicas deve-se à inclusão de medicamentos para doença de Alzheimer na versão de 2012. Por outro lado, atende a menos causas de câncer, o que pode ser reflexo desse novo formato de RENAME, visto que tais medicamentos não fazem parte dos atuais Componentes de Financiamento da Assistência Farmacêutica e da Tabela de Procedimentos, Órteses, Próteses e Medicamentos do SUS da RENASES.

O número de medicamentos com indicações terapêuticas para as 15 principais causas de DALY é semelhante entre as RENAME 2002, 2006 e 2008, havendo pequenas inclusões ou exclusões pautadas na eficácia do medicamento, segurança ou até mesmo na essencialidade do medicamento, como no caso da exclusão da atorvastatina entre as RENAME 2002 e 2006 (Tabela 3).
Na Tabela 4, é possível observar diferenças entre as versões da RENAME 2010 e 2012. A RENAME 2012 indica um número maior de medicamentos para o tratamento de doença cardíaca isquêmica, doença pulmonar obstrutiva crônica (DPOC), doença de Alzheimer, asma, HIV/ AIDS, epilepsia, além de número bem maior para doenças endócrino-metabólicas.

A Tabela 5 apresenta os medicamentos que constam na Rename 2012, para as principais causas de DALY do estudo Carga Global de Doença Brasil 2008, verificando sua presença na lista da OMS e sua eficácia, força de evidência e força de recomendação.

Para doença isquêmica do coração, a RENAME 2012 incluiu três antiagregantes plaquetários (clopidogrel, abciximab e tenecteplase), dois trombolíticos (alteplase e tenecteplase) e ainda quatro estatinas e dois fibratos. Nenhum desses medicamentos se encontra na lista de medicamentos essenciais da OMS 15. Entretanto, o clopidogrel apresenta evidência científica igual à do ácido acetilsalicílico para prevenção 
Tabela 2

Causas de DALY do estudo Carga de Doença do Brasil 2008 atendidas pelas RENAME 2010 e 2012, por grupo de causas.

\begin{tabular}{|c|c|c|c|c|}
\hline \multirow[t]{3}{*}{ Causas (2o nível) } & \multicolumn{4}{|c|}{ RENAME } \\
\hline & \multicolumn{2}{|l|}{2010} & \multicolumn{2}{|l|}{2012} \\
\hline & $\begin{array}{l}\text { Número de causas } \\
\text { atendidas/Número de } \\
\text { causas existentes nos } \\
\text { grupos }\end{array}$ & $\%$ & $\begin{array}{l}\text { Número de causas } \\
\text { atendidas/Número de } \\
\text { causas existentes nos } \\
\text { grupos }\end{array}$ & $\%$ \\
\hline Doenças cardiovasculares & $6 / 6$ & 100 & $6 / 6$ & 100 \\
\hline Doenças aparelho digestivo & $3 / 3$ & 100 & $3 / 3$ & 100 \\
\hline Doenças musculoesqueléticas & $3 / 3$ & 100 & $3 / 3$ & 100 \\
\hline Doenças respiratórias crônicas & $3 / 3$ & 100 & $3 / 3$ & 100 \\
\hline Infecções respiratórias & $3 / 3$ & 100 & $3 / 3$ & 100 \\
\hline Condições durante o período perinatal & $2 / 2$ & 100 & $2 / 2$ & 100 \\
\hline Desordens de órgãos dos sentidos & $2 / 2$ & 100 & $2 / 2$ & 100 \\
\hline Causa externa não intencional & $1 / 1$ & 100 & $1 / 1$ & 100 \\
\hline Diabetes mellitus & $1 / 1$ & 100 & $1 / 1$ & 100 \\
\hline Doenças de pele & $1 / 1$ & 100 & $1 / 1$ & 100 \\
\hline Doenças endócrinas/metabólicas & $1 / 1$ & 100 & $1 / 1$ & 100 \\
\hline Infecciosas e parasitárias & $18 / 20$ & 90 & $19 / 20$ & 95 \\
\hline Doenças neurológicas & $4 / 5$ & 80 & $5 / 5$ & 100 \\
\hline Condições maternas & $4 / 5$ & 80 & $4 / 5$ & 80 \\
\hline Doenças psiquiátricas & $7 / 9$ & 78 & $7 / 9$ & 78 \\
\hline Câncer & $10 / 13$ & 77 & $7 / 13$ & 54 \\
\hline Doenças geniturinárias & $2 / 3$ & 67 & $2 / 3$ & 67 \\
\hline Deficiências nutricionais & $1 / 2$ & 50 & $2 / 2$ & 100 \\
\hline Condições orais & $1 / 2$ & 50 & $1 / 2$ & 50 \\
\hline Total & $73 / 85$ & 86 & $73 / 85$ & 87 \\
\hline
\end{tabular}

DALY: disability adjusted life of years; RENAME: Relação Nacional de Medicamentos Essenciais.

secundária de infarto agudo do miocárdio 16. Já entre os trombolíticos incluídos, nenhum possui evidência semelhante à estreptoquinase.

Para doença pulmonar obstrutiva crônica e para asma, foi incluído um segundo broncodilatador de curta ação (fenoterol). Além de não constar na lista de medicamentos essenciais da OMS 15, o fenoterol não é aprovado nos Estados Unidos, pois pode provocar reações adversas graves como taquiarritmias e morte súbita, especialmente em altas doses e situação de hipoxia 17 (Thomson Reuters Healthcare Community. DrugDex System. http://www.micromedex.com/ products/drugdex/, acessado em 27/Mai/2013). Foram inseridos ainda dois broncodilatadores de longa ação (salmeterol e formoterol). Embora não constantes na lista de medicamentos essenciais da OMS 15, a revisão da RENAME 2010, realizada pela Comissão Técnica e Multidisciplinar de Atualização da Relação Nacional de Medicamentos Essenciais (COMARE), propôs a inclusão do formoterol ou salmeterol para o tratamento de doença pulmonar obstrutiva crônica 10 .

O estudo de Carga Global de Doença classifica como doenças metabólicas uma combinação de diferentes capítulos da CID10 (D55-89; E03-07; E15-E16; E20-E34; E51-89), congregando, dessa forma, distúrbios endócrinos, hematopoiéticos, dislipidemias, síndromes específicas, entre outros. Tal classificação reflete o alto número de medicamentos presentes na RENAME 2010 e o aumento na RENAME 2012. Entre os medicamentos incluídos na RENAME 2012, para doenças endócrinas e metabólicas, destacam-se sete indicados para dislipidemia, sendo quatro estatinas (atorvastatina, fluvastatina, lovastatina, pravastatina) e três fibratos (bezafibrato, ciprofibrato, etofibrato). Dentre esses, apenas a sinvastatina está na lista da OMS 15.

Embora as estatinas incluídas apresentem recomendação semelhante à sinvastatina para pacientes com dislipidemia, a sinvastatina e a ator- 
Número de medicamentos das RENAME 2002, 2006 e 2008 segundo as 15 primeiras causas de DALY do Projeto de Carga de Doença Brasil 1998.

\begin{tabular}{|c|c|c|c|}
\hline \multirow[t]{3}{*}{ Causas (3o nível) } & \multicolumn{3}{|c|}{ RENAME } \\
\hline & 2002 & 2006 & 2008 \\
\hline & $\mathbf{n}$ & $\mathbf{n}$ & n \\
\hline Diabetes mellitus & 4 & 5 & 5 \\
\hline Doença cardíaca isquêmica & 10 & 12 & 12 \\
\hline Acidente vascular cerebral & 2 & 2 & 2 \\
\hline Transtorno depressivo recorrente/Episódio depressivo & 3 & 4 & 4 \\
\hline Doença pulmonar obstrutiva crônica & 4 & 4 & 4 \\
\hline Infecções de vias aéreas inferiores & 20 & 19 & 19 \\
\hline Doença de Alzheimer e outras demências & 0 & 0 & 0 \\
\hline Transtornos mentais e comportamentais devido ao uso de álcool & 3 & 3 & 3 \\
\hline Diarreia e infecções intestinais & 4 & 5 & 6 \\
\hline Esquizofrenia/Psicose & 2 & 2 & 3 \\
\hline Asma & 8 & 6 & 6 \\
\hline Outras causas externas não intencionais & 11 & 10 & 11 \\
\hline Doenças endócrinas/metabólicas* & 28 & 25 & 25 \\
\hline Cirrose - álcool e outras & 3 & 3 & 3 \\
\hline Anemia por deficiência de ferro & 2 & 1 & 1 \\
\hline Total de Medicamentos & 104 & 101 & 104 \\
\hline
\end{tabular}

* Inclui diferentes códigos da Classificação Internacional de Doenças - 10ạ revisão (CID-10): D55-89; E03-07; E15-E16; E20-E34;E51-89.

DALY: disability adjusted life of years; RENAME: Relação Nacional de Medicamentos Essenciais.

vastatina são medicamentos com maiores vantagens, entre as estatinas, na redução do colesterol sérico. Isso porque as outras estatinas, apesar de eficazes, não apresentam vantagens. Ademais, as estatinas, como classe, apresentam maior eficácia frente aos fibratos (Thomson Reuters Healthcare Community. DrugDex System. http://www. micromedex.com/products/drugdex/, acessado em 27/Mai/2013).

A união das listas dos Componentes de Financiamento da Assistência Farmacêutica para constituição da nova RENAME refletiu no aumento de medicamentos para causas já contempladas nas RENAME anteriores, como o caso de medicamentos para HIV/AIDS, por conta do grande número de antirretrovirais constantes no elenco do CESAF. Dos seis antirretrovirais incluídos na RENAME 2012, somente um (indinavir) encontra-se na lista da OMS 15.

Merece destaque ainda o fato de a etravirina e o tipranivir constarem no DrugDex - Thomson Micromedex como medicamentos utilizados em tratamento experimental para pacientes com infecção por HIV (Thomson Reuters Healthcare Community. DrugDex System. http://www. micromedex.com/products/drugdex/, acessado em 27/Mai/2013). Tal fato nos faz questionar a segurança desses medicamentos incluídos na RENAME 2012.

O elenco de medicamentos indicados para epilepsia também dobrou, por causa do número de medicamentos já constantes no elenco do CEAF. Foram incluídos o clobazam, gabapentina, lamotrigina, primidona, topiramato, vigabatrina e etossuximida, mas apenas o último se encontra na lista da OMS 15. Com exceção da etossuximida, os outros medicamentos inseridos possuem recomendação IIb, ou seja, são recomendados em alguns casos.

Vale ressaltar a inclusão dos medicamentos donepezila, galantamina e rivastigmina, indicados para Doença de Alzheimer, mas não encontrados na lista da OMS. Ainda que ensaios clínicos duplo-cego mostrem que esses três inibidores da colinesterase são eficazes para Doença de Alzheimer leve a moderada, por melhorarem a cognição, a função global e atividades da vida diária, ainda há dúvidas em relação à diferença nas taxas de progressão de incapacidade ou institucionalização, quando da utilização desses inibidores da colinesterase no longo prazo 18,19,20,21,22. Ademais, questiona-se a qualidade dos estudos 
Tabela 4

Número de medicamentos das RENAME 2010 e 2012

segundo as 15 primeiras causas de DALY do estudo

Carga de Doença do Brasil 2008.

\begin{tabular}{|c|c|c|}
\hline \multirow[t]{3}{*}{ Causas (3o nível) } & \multicolumn{2}{|c|}{ RENAME } \\
\hline & 2010 & 2012 \\
\hline & $\mathbf{n}$ & $\mathbf{n}$ \\
\hline Depressão & 4 & 4 \\
\hline Doença cardíaca isquêmica & 9 & 20 \\
\hline Diabetes mellitus & 5 & 5 \\
\hline Acidente vascular cerebral & 3 & 3 \\
\hline Doença pulmonar obstrutiva crônica & 5 & 8 \\
\hline Abuso e dependências de álcool & 3 & 3 \\
\hline Transtorno afetivo bipolar & 7 & 7 \\
\hline Infecções de vias aéreas inferiores & 19 & 19 \\
\hline $\begin{array}{l}\text { Doença de Alzheimer e } \\
\text { outras demências }\end{array}$ & 0 & 3 \\
\hline Asma & 4 & 8 \\
\hline Doenças endócrinas e metabólicas * & 25 & 53 \\
\hline Cirrose - álcool e outras & 3 & 3 \\
\hline Doenças cardíacas hipertensivas & 13 & 13 \\
\hline HIV/AIDS & 12 & 18 \\
\hline Epilepsia & 7 & 14 \\
\hline Total de medicamentos & 123 & 182 \\
\hline
\end{tabular}

* Inclui diferentes códigos da Classificação Internacional de Doenças - 10ạ revisão (CID 10): D55-89; E03-07; E15-E16; E20-E34;E51-89.

DALY: disability adjusted life of years; RENAME: Relação Nacional de Medicamentos Essenciais.

que fundamentam a evidência e a relação custo do medicamento e anos de vida ajustados por qualidade 20,23,24.

As listas de medicamentos essenciais buscam dar racionalidade ao uso do medicamento, por meio da eficácia, qualidade e custo-efetividade. A RENAME 2012 atende praticamente às mesmas causas da Carga de Doença do Brasil 2008, quando comparada à RENAME 2010, porém com um número superior de medicamentos. Assim, o aumento no número de medicamentos, sem corresponder ao aumento no número proporcional de causas de DALY contempladas, levanta a discussão acerca da necessidade clínica, uma vez que foram incluídos medicamentos de mesma classe para uma única situação patológica, sem aparente evidência científica.

A diferenciação de produtos como padrão de competição é uma característica do mercado farmacêutico e leva à inserção de inúmeros medicamentos no mercado 25 , sem que isso se traduza em uma melhoria da terapêutica disponibilizada à população 25,26. Em meio a esse contexto de grande oferta de produtos, a RENAME 2012 parece ter sido tensionada tanto por pressões do crescente mercado de novas tecnologias em saúde como pelas oportunidades políticas de ofertar medicamentos, muitas vezes presentes em ações judiciais 27 , deixando de ser uma relação de medicamentos essenciais e se tornando uma lista de financiamento da assistência farmacêutica.

Dentre as vantagens de uma lista limitada de fármacos de elevada prioridade estão: (1) a manutenção de substâncias ineficazes ou tóxicas fora do uso comum; (2) a opção por medicamentos com melhor relação custo-efetividade entre produtos terapeuticamente equivalentes; (3) gerência mais eficiente de um limitado estoque farmacêutico; e (4) a necessidade de produzir informações sobre os fármacos disponíveis somente para um número definido e limitado deles 17. A RENAME 2012 parece ir de encontro ao que é preconizado pela OMS na seleção de medicamentos essenciais, no que tange a utilizar os critérios de eficácia, segurança, conveniência, qualidade e comparação de custo ${ }^{3}$, destacando-se a possível exposição de indivíduos a medicamentos com segurança questionável ou a medicamentos sem efetividade bem definida para algumas indicações terapêuticas. Questiona-se ainda se, na seleção de medicamentos da RENAME 2012, foram ponderados os custos e os benefícios da administração de um novo tratamento em relação a outro já existente.

Diferentemente das versões anteriores, a RENAME 2012 não é acompanhada de apêndices detalhados com justificação de exclusões, inclusões e alterações (apresentação e/ou concentração e/ou dose), assim como informação relacionada à indicação terapêutica e restrição de uso.

A RENAME é o eixo do Ciclo da Assistência Farmacêutica, e é com base nela que as demais atividades (programação, aquisição, armazenamento, distribuição e dispensação) são desenvolvidas. Dessa forma, o aumento do número de medicamentos selecionados pode interferir e dificultar a gestão dos medicamentos essenciais 27,28. Além disso, a RENAME 2012, não sendo uma continuação das versões anteriores, pode gerar desorientação para gestores, prescritores, dispensadores, com provável prejuízo para os usuários do SUS 27.

Por outro lado, destaca-se o uso dos estudos de carga global de doença na construção de políticas de saúde. Grande parte das críticas acerca dos estudos de carga global de doenças centra-se na construção do indicador DALY, particularmente em relação às escolhas sociais em torno dos pesos das incapacidades e dos ajustes. 
Tabela 5

Medicamentos integrantes da RENAME 2012 para as principais causas de DALY do estudo Carga de Doenças do Brasil 2008 , segundo presença na lista de medicamentos essenciais da OMS, eficácia, força de evidência e força de recomendação.

\begin{tabular}{|c|c|c|}
\hline Causas (3o nível)/Medicamentos (RENAME 2012) & OMS & Eficácia, força da evidência e força de recomendação \\
\hline \multicolumn{3}{|c|}{ Doença cardíaca isquêmica (angina e infarto agudo do miocárdio) } \\
\hline Ácido acetilsalicílico * & Sim & Efetivo, classe I, Categoria A \\
\hline Clopidogrel & Não & Efetivo, classe I, Categoria A \\
\hline Abciximabe & Não & Evidência favorece eficácia, classe Ila, Categoria B \\
\hline Cloridrato de tirofibana & Não & Efetivo, classe Ila, Categoria A \\
\hline Estreptoquinase * & $\operatorname{Sim}$ & Efetivo, classe I, Categoria A \\
\hline Alteplase & Não & Efetivo, classe Ila, Categoria A \\
\hline Tenecteplase & Não & Efetivo, classe Ila, Categoria B \\
\hline Atenolol * & Não & Efetivo, classe Ila, Categoria B \\
\hline Propranolol * & $\operatorname{Sim}$ & Efetivo, classe Ila, Categoria B \\
\hline Anlodipino * & $\operatorname{Sim}$ & Efetivo, classe Ila, Categoria A \\
\hline Verapamil * & $\operatorname{Sim}$ & Efetivo, classe Ila, Categoria B \\
\hline Enalapril * & $\operatorname{Sim}$ & Efetivo, classe Ila, Categoria B \\
\hline Losartana & Não & Evidência favorece eficácia, classe Ila, Categoria B \\
\hline Heparina * & $\operatorname{Sim}$ & Evidência favorece eficácia, classe Ila, Categoria B \\
\hline Dinitrato de isossorbida * & $\operatorname{Sim}$ & Efetivo, classe Ila, Categoria B \\
\hline Monoidrato de isossorbida * & Não & Evidência favorece eficácia, classe Ila, Categoria B \\
\hline Sinvastatina * & $\operatorname{Sim}$ & Efetivo, classe Ila, classe Ila, Categoria B \\
\hline Fenofibrato * & Não & Evidência favorece eficácia, classe Ila, Categoria B \\
\hline Atorvastatina & Não & Efetivo, classe Ila, Categoria A \\
\hline Fluvastatina & Não & Evidência favorece eficácia, classe Ila, Categoria B \\
\hline Lovastatina & Não & Evidência favorece eficácia, classe Ila, Categoria B \\
\hline Pravastatina & Não & Evidência favorece eficácia, classe Ila, Categoria B \\
\hline Bezafibrato & Não & Evidência favorece eficácia, classe Ilb, Categoria B \\
\hline Genfibrozila & Não & Efetivo, classe Ilb, Categoria B \\
\hline \multicolumn{3}{|l|}{ Doença pulmonar obstrutiva crônica } \\
\hline Ipratrópio * & Sim & Efetivo, classe Ila, Categoria B \\
\hline Salbutamol * & $\operatorname{Sim}$ & Efetivo, classe Ila, Categoria B \\
\hline Fenoterol & Não & Evidência favorece eficácia, classe Ilb, Categoria B \\
\hline Salmeterol & Não & Efetivo, classe Ila, Categoria B \\
\hline Formoterol & Não & Efetivo, classe Ila, Categoria B \\
\hline \multicolumn{3}{|l|}{ Doença de Alzheimer e outras demências } \\
\hline Galantamina & Não & Efetivo, classe Ila, Categoria B \\
\hline Rivastigmina & Não & Efetivo, classe Ila, Categoria B \\
\hline Donepezila & Não & Efetivo, classe Ila, Categoria B \\
\hline \multicolumn{3}{|l|}{ Asma } \\
\hline Beclometasona * & $\operatorname{Sim}$ & Efetivo, classe I, Categoria B \\
\hline Budesonida * & $\operatorname{Sim}$ & Efetivo, classe Ila, Categoria B \\
\hline Prednisolona * & $\operatorname{Sim}$ & Efetivo, classe Ila, Categoria B \\
\hline Salmeterol & Não & Efetivo, classe Ilb, Categoria B \\
\hline Formoterol & Não & Efetivo, classe Ila, Categoria B \\
\hline Salbutamol * & $\operatorname{Sim}$ & Efetivo, classe I, Categoria B \\
\hline Fenoterol & Não & Evidência favorece eficácia, classe Ilb, Categoria B \\
\hline
\end{tabular}

(continua) 


\begin{tabular}{|c|c|c|}
\hline Causas (3o nível)/Medicamentos (RENAME 2012) & OMS & Eficácia, força da evidência e força de recomendação \\
\hline \multicolumn{3}{|l|}{ HIV/AIDS } \\
\hline Abacavir * & Sim & Efetivo, classe Ilb, Categoria A \\
\hline Didanosina * & Sim & Efetivo, classe Ilb, Categoria A \\
\hline Entecavir & Não & Efetivo, classe Ila, Categoria B \\
\hline Estavudina & Sim & Efetivo, classe Ilb, Categoria B \\
\hline Lamivudina * & Sim & Efetivo, classe Ila, Categoria A \\
\hline Zidovudina * & Sim & Efetivo, classe Ilb, Categoria A \\
\hline Zidovudina+lamivudina * & Sim & Efetivo, classe Ilb, Categoria B \\
\hline Efavirenz * & Sim & Efetivo, classe Ila, Categoria A \\
\hline Etravirina & Não & Evidência favorece eficácia, classe Ilb, Categoria B \\
\hline Tenofovir * & $\operatorname{Sim}$ & Efetivo, classe Ila, Categoria B \\
\hline Nevirapina * & Sim & Efetivo, classe Ilb, Categoria A \\
\hline Lopinavir+ritonavir * & Sim & Efetivo, classe Ila, Categoria B \\
\hline Ritonavir * & $\operatorname{Sim}$ & Efetivo, classe Ila, Categoria B \\
\hline Saquinavir * & Sim & Efetivo, classe Ila, Categoria A \\
\hline Atazanavir * & Sim & Evidência favorece eficácia, classe Ila, Categoria A \\
\hline Darunavir & Não & Efetivo, classe Ila, Categoria B \\
\hline Fosamprenavir & Não & Efetivo, classe Ila, Categoria B \\
\hline Indinavir & Sim & Efetivo, classe Ilb, Categoria A \\
\hline Tipranavir & Não & Evidência favorece eficácia, classe Ilb, Categoria B \\
\hline Enfuvirtida & Não & Efetivo, classe Ila, Categoria B \\
\hline Raltegravir & Não & Efetivo, classe Ila, Categoria B \\
\hline \multicolumn{3}{|l|}{ Epilepsia } \\
\hline Clobazam & Não & Evidência favorece eficácia, classe III, Categoria B \\
\hline Etossuximida & Sim & Efetivo, classe Ila, Categoria B \\
\hline Gabapentina & Não & Efetivo, classe Ilb, Categoria B \\
\hline Lamotrigina & Não & Evidência favorece eficácia, classe Ila, Categoria B \\
\hline Primidona & Não & Evidência favorece eficácia, classe Ilb, Categoria B \\
\hline Topiramato & Não & Efetivo, classe Ilb, Categoria B \\
\hline Carbamazepina * & Sim & Efetivo, classe Ila, Categoria B \\
\hline Clonazepam * & Sim & Efetivo, classe Ila, Categoria B \\
\hline Diazepam * & Sim & Efetivo, classe Ila, Categoria B \\
\hline Sulfato de magnésio* & Sim & Efetivo, classe Ila, Categoria B \\
\hline Valproato de sódio ou ácido valpróico * & Sim & Efetivo, classe Ila, Categoria B \\
\hline Fenitoína * & Sim & Efetivo, classe Ila, Categoria B \\
\hline Fenobarbital * & Sim & Efetivo, classe Ila, Categoria B \\
\hline Vigabatrina & Não & Evidência favorece eficácia, classe IIB, Categoria B \\
\hline \multicolumn{3}{|l|}{ Dislipidemia } \\
\hline Sinvastatina * & Sim & Efetivo, classe Ila, Categoria A \\
\hline Fenofibrato * & Não & Efetivo, classe Ila, Categoria B \\
\hline Atorvastatina & Não & Evidência favorece eficácia, classe Ila, Categoria B \\
\hline Fluvastatina & Não & Efetivo, classe Ila, Categoria B \\
\hline Lovastatina & Não & Efetivo, classe Ila, Categoria B \\
\hline Pravastatina & Não & Efetivo, classe Ila, Categoria A \\
\hline Bezafibrato & Não & Evidência favorece eficácia, classe III, Categoria B \\
\hline Genfibrozila & Não & Efetivo, classe Ilb, Categoria B \\
\hline Ciprofibrato & Não & Evidência favorece eficácia, classe III, Categoria B \\
\hline Etofibrato & Não & Evidência favorece eficácia, classe III, Categoria B \\
\hline
\end{tabular}

* Medicamentos integrantes da RENAME 2010.

DALY: disability adjusted life of years; OMS: Organização Mundial da Saúde; RENAME: Relação Nacional de Medicamentos Essenciais. 
Contudo, medidas epidemiológicas, como incidência e prevalência, por mais que sejam uma importante fonte de informação utilizada para identificar problemas de saúde na população, deixam de lado elementos complexos, podendo levar a inconsistências importantes 14,29,30. O DALY é uma medida única que permite medir a magnitude de uma determinada doença no óbito prematuro e as morbidades com suas respectivas sequelas, que, por sua vez, comprometem a qualidade de vida dos indivíduos 14 . Nesse sentido, o DALY tem sido utilizado como uma ferramenta para definição de prioridades no setor da saúde, auxiliando na construção das políticas enquanto instrumento de avaliação do desempenho dos sistemas e para avaliação de pesquisas em saúde 31,32 .

\section{Considerações finais}

Na seleção de medicamentos essenciais, a OMS orienta a utilização da carga global de doença para a identificação dos problemas de saúde da população. As versões analisadas da RENAME atendem de forma satisfatória à carga global de doença no Brasil, mesmo havendo modificações na metodologia de classificação das causas entre os estudos de Carga Global de Doença 1998 e 2008, configurando-se como uma limitação do presente trabalho.

Outra limitação é a classificação dos medicamentos segundo causa de DALY dos estudos de carga global de doença, sobretudo para medicamentos com mais de uma indicação terapêutica, como no caso dos antibióticos. Assim, buscando melhorar a classificação, foram consultadas diversas bases e, por fim, um especialista.
As RENAME 2002, 2006 e 2008 atenderam igualmente à Projeto Carga de Doença 1998, sendo semelhantes ainda no número de medicamentos. Tal fato demonstra que as modificações entre as versões, inclusões, ou até mesmo pequenas modificações na apresentação dos medicamentos integrantes levaram essas versões a manter semelhante espectro de atuação terapêutica. Por outro lado, ainda que as RENAME 2010 e 2012 atendam praticamente às mesmas causas da Carga de Doença do Brasil 2008, a versão de 2012 o faz com um número superior de medicamentos. A RENAME 2012, ao representar a união dos elencos de medicamentos dos Componentes de Financiamento da Assistência Farmacêutica, faz supor que a maior diferença entre a RENAME 2012 e as demais esteja no critério de essencialidade. Os medicamentos dos Componentes buscam, em alguns casos, atender às particularidades da população por eles coberta, o que vai de encontro ao conceito de medicamentos essenciais preconizado pela OMS.

Em resumo, o aumento do número de medicamentos não correspondeu ao aumento do número de causas cobertas. Por conseguinte, a identificação de algumas causas, como a dislipidemia, epilepsia e HIV/AIDS, para as quais o número de medicamentos cresceu desmesuradamente, aponta para a necessidade de uma análise mais aprofundada, que possa verificar se esse aumento se faz de maneira a favorecer o uso racional de medicamentos e subsidiar as demais diretrizes da Política Nacional de Medicamentos.

Assim sendo, recomenda-se a realização de novos estudos que relacionem os fármacos selecionados no Brasil para a Lista de Medicamentos Essenciais, bem como seus Protocolos Clínicos e Diretrizes Terapêuticas (PCDT), com as listas e PCDT de outros países, que tenham realizado estudo de carga global de doença. 


\section{Resumen}

Los medicamentos esenciales son definidos por la Organización Mundial de la Salud (OMS) como aquellos que satisfacen las necesidades de atención de salud prioritarias de la población. Su selección tiene como objetivo reflejar las necesidades colectivas, recomendando, por lo tanto, el uso de estudios de carga global de enfermedades. Se realizó un estudio exploratorio que une el fármaco a los estudios de cambios de nombres en la Carga Global de Enfermedad de Brasil, 1998 y 2008, y evidencia científica. Buscamos, por lo tanto, verificar que la relación de los medicamentos en RENAME (2002-2012) cumplen las recomendaciones de la OMS para la selección de medicamentos. Pese a que RENAME 2010 y 2012 se sitúan de manera similar por la Carga Global de Enfermedad de Brasil 2008, la versión 2012 incluye más medicamentos, lo que se ha producido por las presiones en el crecimiento del mercado de las nuevas tecnologías. Así, el RENAME, ya no es una relación de los medicamentos esenciales y ha pasado a convertirse en una lista de financiación para la atención farmacéutica.

Medicamentos Esenciales; Carga de la Enfermedad; Servicios Farmaceúticos

\section{Colaboradores}

T. A. Figueiredo contribuiu com a concepção, realização das análises, redação e revisão do artigo. J. M. A. Schramm e V. L. Pepe contribuíram com a concepção, redação e revisão do artigo.

\section{Referências}

1. Ministério da Saúde. Portaria no 3.916 de 10 de novembro de 1998. Aprova a Política Nacional de Medicamentos. Diário Oficial da União 1990; 10 nov.

2. Conselho Nacional de Saúde. Resolução no 338 de 6 de maio de 2004. Aprova a Política Nacional de Assistência Farmacêutica. Diário Oficial da União 2004; 20 mai.

3. World Health Organization. The world medicines situation 2011 - selection of essential medicines. (acessado em 10/Dez/2012).

4. Schramm JMA, Oliveira AF, Leite IC, Valente JG, Gadelha AMJ, Portela MC, et al. Transição epidemiológica e o estudo de carga de doença no Brasil. Ciênc Saúde Coletiva 2004; 9:897-908.

5. Gadelha C. Perspectivas do investimento em saúde. Rio de Janeiro: Universidade Federal do Rio de Janeiro; 2009 .

6. Wannmacher L. Medicamentos essenciais: vantagens de trabalhar com este contexto. Uso Racional de Medicamentos: Temas Selecionados 2005; 3:1-6.

7. Hogerzeil HV. The concept of essential medicines: lessons for rich countries. BMJ 2004; 329:1169-72.
8. Sant'Ana JMB, Pepe VLE, Osorio-de-Castro CGS, Ventura M. Essencialidade e assistência farmacêutica: considerações sobre o acesso a medicamentos mediante ações judiciais no Brasil. Rev Panam Salud Pública 2011; 29:138-44.

9. Messeder AM, Osorio-de-Castro CGS, Luiza VL. Mandados judiciais como ferramenta para garantia do acesso a medicamentos no setor público: a experiência do Estado do Rio de Janeiro, Brasil. Cad Saúde Pública 2005; 21:525-34.

10. Departamento de Assistência Farmacêutica e Insumos Estratégicos, Secretaria de Ciência, Tecnologia e Insumos Estratégicos, Ministério da Saúde. Relação Nacional de Medicamentos Essenciais RENAME 2010. Brasília: Ministério da Saúde; 2010. (Série B. Textos Básicos de Saúde).

11. Departamento de Assistência Farmacêutica e Insumos Estratégicos, Secretaria de Ciência, Tecnologia e Insumos Estratégicos, Ministério da Saúde. Relação Nacional de medicamentos essenciais RENAME. Brasília: Ministério da Saúde; 2012. (Série B. Textos Básicos de Saúde). 
12. Ministério da Saúde. Portaria no 841 , de 2 de maio de 2012. Publica a Relação Nacional de Ações e Serviços de Saúde (RENASES) no âmbito do Sistema Único de Saúde (SUS) e dá outras providências. Diário Oficial da União 2012; 2 mai.

13. World Health Organization. The global burden of disease - 2004. http://www.who.int/healthinfo/ global_burden_disease/2004_report_update/en/ index.html (acessado em 08/Mar/2012).

14. Capellà D. Descritive tools and analysis. In: Dukes MNG, editor. Drug utilization studies: methods and uses. Copenhagen: World Health Organization Regional Publications; 1993. p. 55-78. (European Series, 45).

15. World Health Organization. WHO model list of essential medicines. 17th list. http://apps.who.int/ iris/bitstream/10665/70640/1/a95053_eng.pdf (acessado em 20/Abr/2013).

16. National Institute for Clinical Excellence. Clopidogrel in the treatment of non-ST-segment-elevation acute coronary syndrome. Technology Appraisal 80. http://www.nice.org.uk/TA080guidance (acessado em 25/Jun/2013).

17. Bonfim JRA, Magalhaes PC. Rename 2012: avanço ou retrocesso para a difusão de medicamentos essenciais? Boletim Farmaco Terapêutica 2013; XVII:9-15.

18. Birks J. Cholinesterase inhibitors (ChEIs), donepezil, galantamine and rivastigmine are efficacious for mild to moderate Alzheimer's disease. http://sum maries.cochrane.org/CD005593/cholinesteraseinhibitors-cheis-donepezil galantamine-and-riv astigmine-are-efficacious-for-mild-to-moderatealzheimers-disease (acessado em 30/Mar/2013).

19. Courtney C1, Farrell D, Gray R, Hills R, Lynch L, Sellwood E, et al. Long-term donepezil treatment in 565 patients with Alzheimer's disease (AD2000): randomized double-blind trial; AD2000 Collaborative Group. Lancet 2004; 363:2105-15.

20. Department of Health. Living well with dementia: a national dementia strategy. http://www. dh.gov.uk/en/Publicationsandstatistics/Publica tions/PublicationsPolicyAndGuidace/DH_094058 (acessado em 16/Mar/2011).

21. Rogers SL, Friedhoff LT. The efficacy and safety of donepezil in patients with Alzheimer's disease: results of a US Multicentre, Randomized, DoubleBlind, Placebo-Controlled Trial. The Donepezil Study Group. Dementia 1996; 7:293-303.
22. Rogers SL, Doody R, Mohs R. E2020 produces both clinical global cognitive test improvement in patients with moderately severe Alzheimer's disease: results of a 30-week phase III trial. Neurology 1996; 46:A217.

23. Reichman WE. Current pharmacologic options for patients with Alzheimer's disease. Ann Gen Hosp Psychiatry 2003; 2:1.

24. Kmietowicz Z. NICE proposes to withdraw Alzheimer's drugs from NHS. BMJ 2005; 330:495.

25. Bastos VD. Inovação farmacêutica: padrão setorial e perspectivas para o caso brasileiro. BNDES Setorial 2005; 22:271-96.

26. Machado-dos-Santos SC. Busca da equidade no acesso aos medicamentos no Brasil: os desafios impostos pela dinâmica da competição "extrapreço" [Dissertação de Mestrado]. Rio de Janeiro: Escola Nacional de Saúde Pública, Fundação Oswaldo Cruz; 2001.

27. Santos-Pinto CDB, Ventura M, Pepe VLE, Osoriode-Castro CGS. Essential medicines and technology incorporation following novel Brazilian Public Health System regulations. Cad Saúde Pública 2013; 29:1056-8.

28. Reis ALA. Novos produtos no mercado farmacêutico: padrão de difusão e preços. Rio de Janeiro Escola Nacional de Saúde Pública, Fundação Oswaldo Cruz; 2004.

29. McKenna MT, Michaud CM. Assessing the burden of disease in the United States using disability-adjusted life years. Am J Prev Med 2005; 28:415-23.

30. Begg SJ, Vos T. Burden of disease and injury in Australia in the new millennium: measuring health loss from diseases, injuries and risk factors. Med J Aust 2008; 188:36-40.

31. Norman R, Matzopoulos R. The high burden of injuries in South Africa. Bull World Health Organ 2007; 85:695-702.

32. Dantés EA. La carga de la enfermedad en países de América Latina. Salud Pública Méx 2011; 53 Suppl 2:S72-7.

Recebido em 17/Set/2013

Versão final reapresentada em 25/Mar/2014

Aprovado em 01/Abr/2014 